\title{
Reliability Analysis of Photovoltaic charging Station of Electric vehicle considering the relationship between Photovoltaic output and charging load
}

\author{
Li Hengjie ${ }^{1,2}$ Cao Xihuan ${ }^{1}$ Li Hong ${ }^{2, *}$ Ji Qingchun ${ }^{3}$ Xu Jianrong ${ }^{3}$ Yue Wenhua ${ }^{3}$ \\ ${ }^{1}$ School of Electrical Engineering and Information Engineering, Lanzhou University of Technology, Lanzhou 730050 \\ ${ }^{2}$ National Experimental Teaching demonstration Center of Electrical and Control Engineering of Lanzhou University of Technology \\ ${ }^{3}$ Lanzhou Power Supply Bureau of Gansu Electric Power Company)
}

\begin{abstract}
The intermittency and randomicity of photovoltaic output will have a great influence on the reliability of photovoltaic charging station. Based on the photovoltaic output and charging load data of a photovoltaic charging station in our country, the reliability of photovoltaic charging station is evaluated. Firstly, the probability distributions of photovoltaic output and charging load are calculated respectively by using nonparametric kernel density estimation method. Secondly, the Copula function is optimized according to the correlation degree. Finally, a hybrid Copula function is constructed to describe the correlation between PV output and EV charging load, and an example is given to verify the reliability of the PV charging station.
\end{abstract}

\section{Introduction}

Photovoltaic power generation plays a vital role in the development and utilization of renewable energy. The output of EV photovoltaic charging station is stochastic and intermittent, and it also has typical probability distribution characteristics. The distributed photovoltaic output and the load of the charging station change with time, which is not only affected by the light, seasonal climate, geography, time and other objective conditions, but also restricted by the charging load of the EV charging station.

Copula function can not only be used to solve complex joint distribution problems with high dimensions, but also can be used to describe multicoupling, multi-influencing factors, nonlinear and asymmetric correlations among variables. Therefore, the Copula function is widely used in the modeling of distributed energy sources. At the same time, the Copula function can also provide a new way to study the correlation between the power output and charging load of EV photovoltaic charging stations. In this paper, the hybrid Copula function is applied to the joint probability distribution model of the output and charging load of photovoltaic generating units. The best copula function was selected according to the fitting degree. Finally, based on the joint probability density function, the reliability of PV charging station is analyzed and evaluated, and its effectiveness is verified by an example.

\section{Probability distribution of photovoltaic output and charging load}

Most of the scholars at home and abroad construct the distributed edge probability model of photovoltaic output based on the solar photovoltaic output probability model. The probability model of radiation distribution can be obtained by using optical radiation data to estimate parameters. Most studies have found that photovoltaic output mostly matches Weibull, Value (Type I), Beta and Norma distributions. But the probability model of deterministic parameter distribution has obvious shortcoming. The distribution parameter needs to be determined according to the prior empirical knowledge. This kind of assumption is done according to the subjective factors, the accuracy of the model will not be quantified, and the parameter model suitable for different areas may not have general applicability. So based on the measured output data of an EV photovoltaic charging station in northwest area, the photovoltaic output and charging load rate of the charging station are selected as random variables to normalize the measured data. A test method based on nonparametric kernel density calculation is proposed by graphing sample data.

The theory of kernel density estimation is based on the frequency histogram. The basic feature of nonparametric kernel density estimation is that it only considers the characteristics of the data itself, and there is no error caused by parameter estimation. A datadriven reliability index can be used to estimate the 
probability density. It does not require a priori knowledge about the form of the probability distribution. This is a method to study the characteristics of the distribution of the data from the point of view of the data samples.

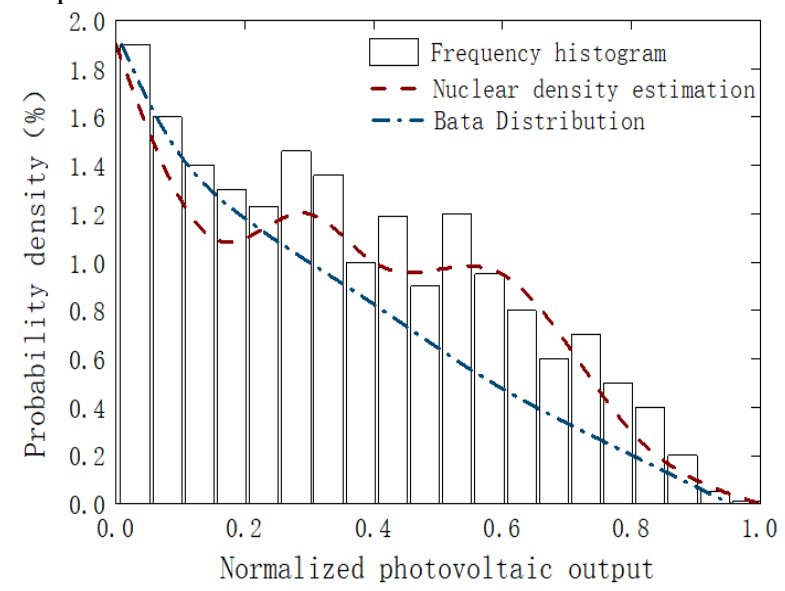

Figure. 1 probability distribution curve of PV output

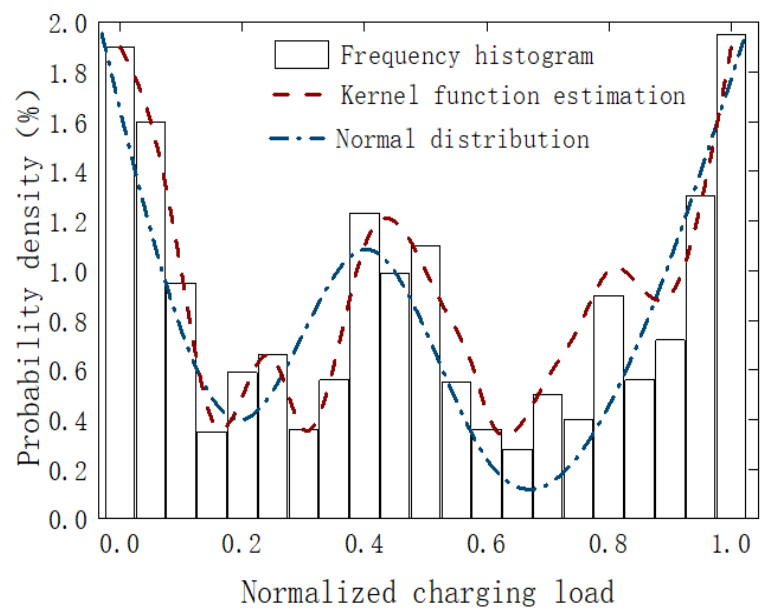

Figure. 2 probability distribution curve of Charge load

\section{Copula Function correlation modeling}

\subsection{Copula Function}

The Copula application of the method is to decompose the joint distribution between variables into the correlation between variables and the edge distribution of a single variable. The application of Copula theory is based on Sklar theorem.

From Sklar Starting from the theorem, the process of constructing Copula function is mainly divided into three steps: one is to construct the edge distribution of each variable; the other is to find the appropriate Copula function and solve its parameters to describe the correlation between the variables; the third is to detect and evaluate the fitting effect of the joint distribution function.

In order to select the appropriate Copula function more intuitively, the actual historical photovoltaic output and charging load data of one year in a northwestern region of China are taken as samples. Because of the fuzzy classification of the sample data, the Gaussian Hybrid Model (GMM) is now used to maximize the expected clustering (EM). The nonparametric kernel density estimation probability distribution of photovoltaic output and load has been obtained according to the measured sample data. On this basis, the relevant parameters of several common Copula functions are estimated by using the Maximum Expectation (EM) clustering algorithm. The EM algorithm is an iterative algorithm for estimating the maximum likelihood of parameters, which has important application in processing "incomplete data". It is emphasized that the EM algorithm can improve the value of the postobservation probability density function after each iteration. The clustering results are shown in Fig. 5. The sample data are divided into two types near the 0.5 intersection line of the two variables. Generating photovoltaic output and load samples with strong correlation. Copula function is used to fit the data after clustering segmentation.

Based on the theory mentioned above, the Copula function with the smallest square Euclidean distance is chosen as a tool to describe the correlation between variables. In the first part, Frank-Copula function is selected to fit the clustered sample data, and in the second part, Clayton-Copula function is selected. Compared with the fitting results before clustering, the proposed clustering method has shorter square Euclidean distance and higher fitting precision.

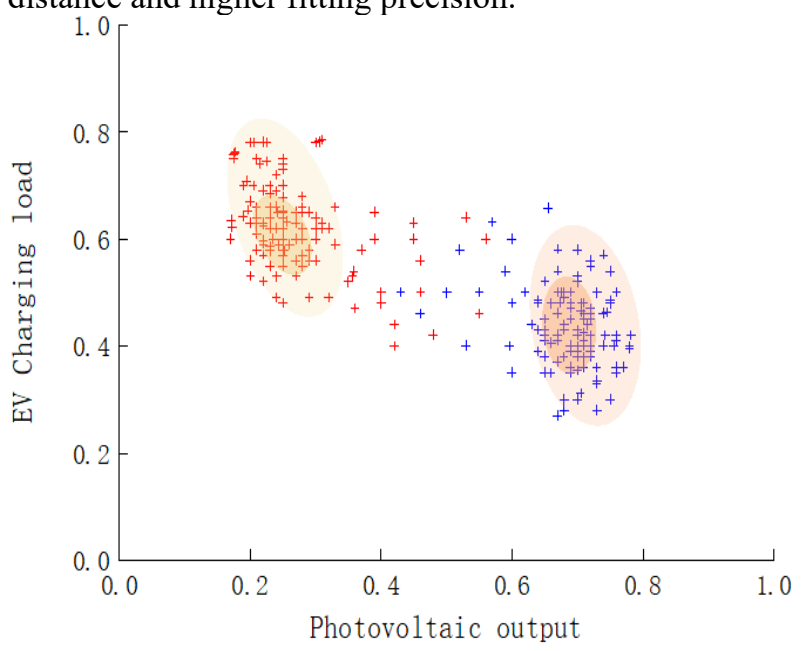

Figure.3 Historical Photovoltaic output and load data EM Clustering

\subsection{Correlation model}

The correlation between photovoltaic output and charging load shown in Fig.4 shows that the two variables have asymmetric rear-tail characteristics. According to the optimal combination of GumbelCopula and Clayton-Copula selected by fitting check, the mixed Copula function is constructed to fit, and the joint probability density function of variable correlation is established, as follows:

$$
C(u, v, \theta)=\omega_{1} C_{G}\left(u, v ; \theta_{1}\right)+\omega_{2} C_{C}\left(u, v ; \theta_{2}\right)
$$




$$
\begin{gathered}
C_{G}\left(u, v, \theta_{1}\right)=\exp \left\{-\left[\left(-\ln (u)^{\theta_{1}}+\left(-\ln (v)^{\theta_{1}}\right]^{\frac{1}{\theta_{1}}}\right\}\right.\right. \\
C_{c}\left(u, v, \theta_{2}\right)=\left(u^{-\theta_{2}}+v^{-\theta_{2}}-1\right)^{-\frac{1}{\theta_{2}}}
\end{gathered}
$$

In formula, $u=F_{p v}\left(P_{p v}\right), v=F_{e v}\left(P_{e v}\right), \omega_{1} 、 \omega_{2}$ are the weight coefficient of the Copula function, and $\omega_{1}+\omega_{2}=1 . \theta_{1} 、 \theta_{2}$ are the linear correlation parameters of the Gumbel-Copula function and the Clayton-Copula. The parameters of this paper are as follows: $\omega_{1}=0.3038, \omega_{2}=0.6962$. The joint probability density is obtained.The distribution is as shown in the Fig.4.

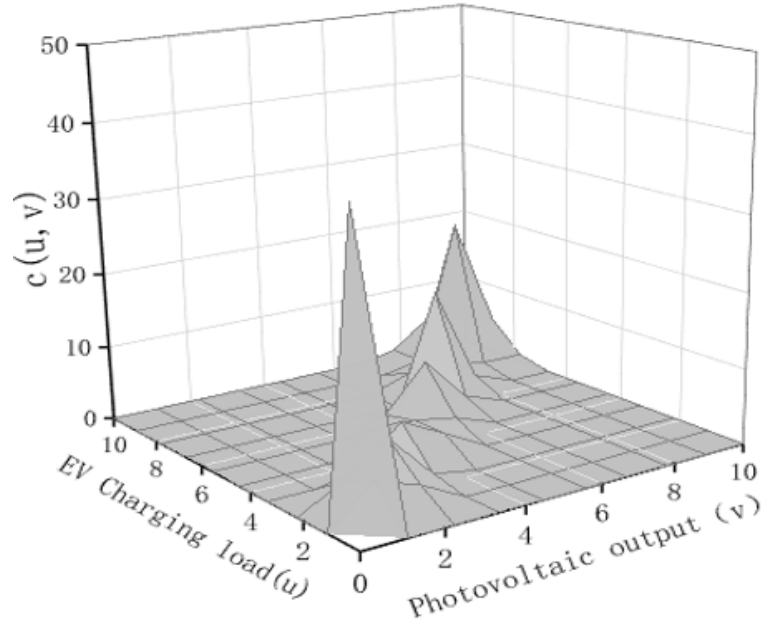

Figure 4. Joint probability density diagram

\section{Example analysis}

\subsection{Reliability assessment indicators and related factors}

In the analysis of an example, the influence of energy storage on reliability needs to be taken into account, and energy storage plays an important role in calming light fluctuation. Secondly, each output resource is serialized with the charging load. The photovoltaic output and energy storage equivalent output are set as resource 1 and resource 2 , respectively. Set the charging load as demand 1 and the equivalent load of energy storage as demand 2. In this paper, the reliability indexes related to margin include the expected value of electricity shortage EENS (Energy Expectation Not Supplied) and the probability of electricity shortage LOLP (Loss of Load Probability).

\subsection{Parameter settings}

The electric vehicle charging station in the example is equipped with 60 , the total capacity of generating set is $1400 \mathrm{MW}$, annual peak charging load $1150 \mathrm{MW}$, to ensure the accuracy of system reliability index calculation, so select the simulation time as a unit for 10 years. The maximum power output of photovoltaic array used in photovoltaic power station is $280 \mathrm{~W}$ under standard test conditions, and the rated capacity of energy storage is $100 \mathrm{MW} . \mathrm{h}$, and the rated power of $100 \mathrm{MW}$, discharge depth is $80 \%$. The value of reliability index EENS is 97.6 MWh.

\subsection{Influence of Photovoltaic output and load correlation on Reliability Evaluation}

The sequential Monte Carlo simulation method of direct sampling is used to explore the influence of photovoltaic output change on the reliability of the system. Tab.1 shows the two groups of data of system reliability index EENS and LOLP, with or without energy storage. It can be seen that the reliability indexes EENS and LOLP increase by $3.23 \%$ and $4.43 \%$ respectively when there is no energy storage in the system, that is to say, the reliability is higher when there is energy storage in the system. Energy storage plays a role in calming light fluctuations, making the output of the joint system more stable, and improving the reliability of the system.

Table 1. The impact of energy storage on system reliability index

\begin{tabular}{|c|c|c|}
\hline Indicators & Energy storage & No energy storage \\
\hline EEN (MW) & 0.0195 & 0.0280 \\
\hline LOLP & 0.0001932 & 0.000243 \\
\hline
\end{tabular}

According to the Copula obtained above, considering the correlation of photovoltaic load. The mixed function parameter model is used to simulate the photovoltaic output and load data by using Copula function EM cluster sampling method. Finally, the EENS and LOLP values of the system reliability index under the two methods are calculated respectively. Finally, the variation of reliability index EENS and LOLP with the increase of photovoltaic power station capacity is calculated in two ways for the example system.

The calculation results are shown in Fig.5.The results show that the values of reliability index LOLP are high at first and then decrease gradually, and finally tend to be stable with the increase of capacity. Because the Copula function model takes into account the correlation between photovoltaic output and load, with the increase of photovoltaic power station capacity, the value of LOLP is always smaller than that of Monte Carlo simulation direct sampling method. Also in Fig.6, the reliability index EENS calculated by parameter fitting direct sampling method is optimistic. It is concluded that the direct sampling method does not consider the correlation between photovoltaic output and load, and the sample data of output power is only simply added with the increase of photovoltaic installed capacity. Obviously, based on Copula mixed function method, after considering the influence of photovoltaic output and load correlation, the simulation results are closer to the reality, and the parameters calculated based on the 
method proposed in this paper are more in line with the actual situation.

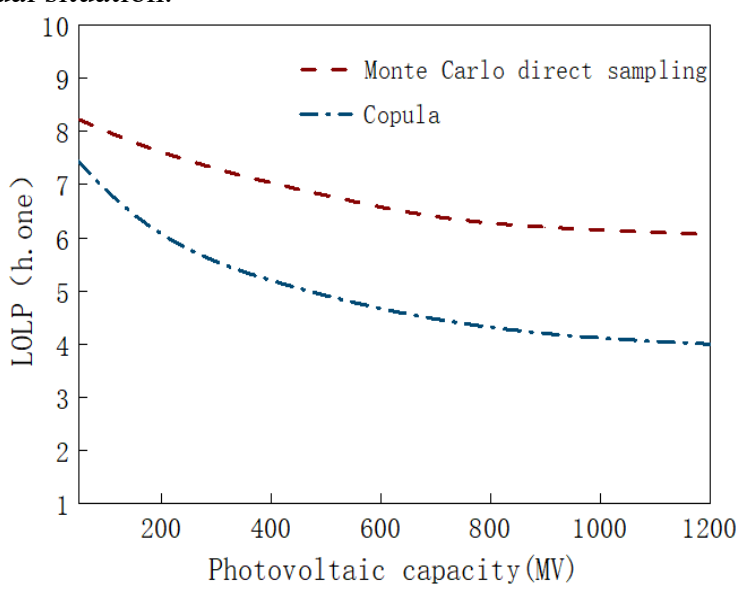

Figure.5 Correlation Model for LOLPThe influence of the value

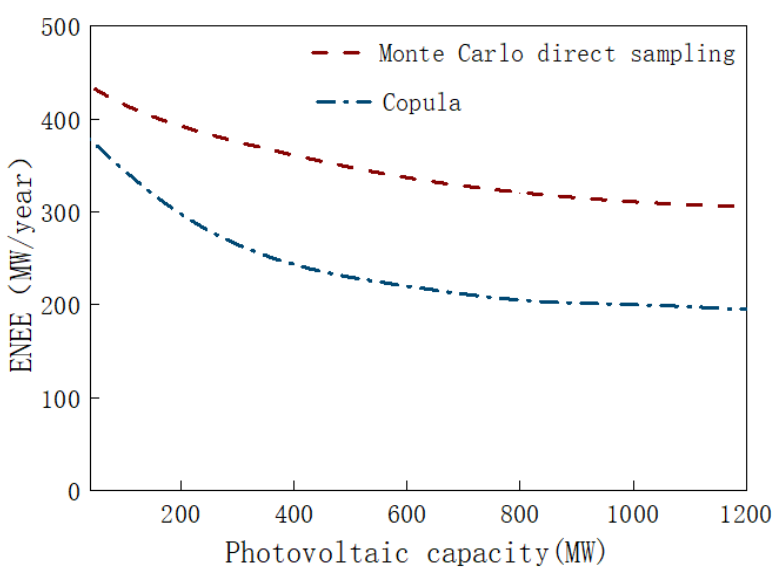

Figure. 6 Correlation Model for ENEEThe influence of the value

\section{Conclusion}

Considering the randomness of photovoltaic output and charging load, the combined probability density model of photovoltaic output and charging load is constructed by using Copula theory. The following conclusions can be drawn by modeling the relativity between photovoltaic output and charging load and verifying and analyzing the examples.

1) The models of photovoltaic output and charging load are established respectively. Instead of the former models of photovoltaic charging station output based on the probability characteristics of solar radiation, the probability distribution obtained by nonparametric kernel density method without prior knowledge is used to improve the accuracy of the model to a great extent.
2) When selecting the mixed Copula function, we should consider the distribution of the sample data and select the best function according to the goodness of fit test method. The fitting test method uses EM clustering algorithm to cluster the data and chooses the best Copula function according to the fitting degree.

3) By modeling the correlation between the photovoltaic output and the charging load in this paper, the reliability indexes related to the margin, including EENS and LOLP, are used to verify the model.

\section{References}

1. Jiang Hao, Lin Shunjiang, Lu Yi, et al.load probability modeling and scene generation of electric vehicle charging station considering time correlation [J] .Electric Power Construction, 2020, 41 (02): 47-57.

2. Wang Hongkun, GE LeiJiao, Li Hongwei, et al. Summary of characteristic analysis and prediction methods for distributed photovoltaic power generation [J]. Electric Power Construction, 2017, 38 (07): 1-9.

3. Yan Wei, Ren Zhouyang, Zhao Xia, et al. Nonparametric nuclear density estimation model of photovoltaic power output power [J]. Power system Automation, 2013, 37 (10): 35-40.

4. Zhao Yuan, Shen Zhijian, Zhou Niancheng, et al. Reliability evaluation of large power grid based on sequential simulation and nonparametric nuclear density measurement. Power system Automation, 2008, 32 (6): 14-19.

5. Li Jing hua, Wen Jin yu, Cheng Shi jie, et al. scene generation method considering Copula correlation of multi-wind farm output [J].Proceedings of the CSEE, 2013, 33 (16): 30-36 $\leq 21$.

6. Luo Yi, Wang Long jun, Wang Gang. Reliability evaluation of microgrid power generation system considering the correlation of distributed power supply output [J] .Power system Automation, 2014, 38 (12): $34-38 \leq 80$.

7. Wang Min, Zong Xianjun, Yuan Yue, et al. Reliability analysis of power generation system including photovoltaic power station [J]. China Electric Engineering Journal, 2013, 33 (34): 42-49 $\leq$ 10.

8. Jin Liming, Zhou Ning, Feng Li, et al.Research on time series probability model of photovoltaic power generation system [J] .Electric Power Construction, 2016, 37 (07): 27-32. 